\title{
Exploiting non-orthogonal multiple access in device-to-device communication
}

\author{
Sang Hoon Lee, Soo Young Shin \\ Department of IT Convergence Engineering, Kumoh National Institute of Technology, South Korea
}

\begin{tabular}{|c|c|}
\hline Article Info & ABSTRACT \\
\hline Article history: & \multirow{11}{*}{$\begin{array}{l}\text { This paper proposes an uplink non-orthogonal multiple access (NOMA) } \\
\text { system with device-to-device (D2D) communication, enabling NOMA users } \\
\text { to communicate with other users/devices using D2D communication to } \\
\text { improve the system capacity. In the NOMA-D2D system, two cellular users } \\
\text { communicated with the BS using uplink NOMA, and two cellular users } \\
\text { simultaneously communicated with the D2D users using downlink NOMA. } \\
\text { Closed-form solutions for the ergodic sum capacity of the proposed system } \\
\text { are derived analytically. The analytical results are validated via simulations } \\
\text { and they are compared with the results obtained from conventional schemes. } \\
\text { The comparison shows that, in scenarios where efficient interference } \\
\text { cancellation can be achieved, the proposed NOMA system with the D2D } \\
\text { model can achieve higher capacity gains than conventional benchmark } \\
\text { schemes. When } \rho=20 \mathrm{~dB} \text {, NOMA-D2D achieves capacity gains of } 192.2 \% \\
\text { and } 157.5 \% \text { over the conventional OMA and the time-sharing-based NOMA, } \\
\text { respectively. }\end{array}$} \\
\hline Received Oct 22, 2020 & \\
\hline Revised Mar 24, 2021 & \\
\hline Accep & \\
\hline Keywords: & \\
\hline Device-to-device & \\
\hline Ergodic sum capacity & \\
\hline Non-orthogonal multiple access & \\
\hline Successive interference & \\
\hline & \\
\hline & \\
\hline
\end{tabular}

This is an open access article under the $\underline{C C B Y-S A}$ license.

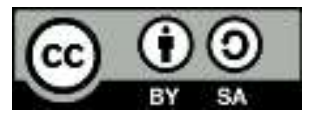

\section{Corresponding Author:}

Soo Young Shin

Wireless and Emerging Network System (WENS) Laboratory

Department of IT Convergence Engineering

Kumoh National Institute of Technology, Gumi, South Korea, 39711

Email:wdragon@kumoh.ac.kr

\section{INTRODUCTION}

Owing to the recent focus on the design of next-generation wireless communication systems and the pursuit to achieve high spectral efficiency in such systems, non-orthogonal multiple access (NOMA) has attracted significant research interest [1]-[7]. According to the NOMA principle, multiple users can communicate over the same resource block (RB), which can be a time slot, a frequency subcarrier, or a scrambling code depending on the context. This sharing process significantly enhances the spectral efficiency of the system [8]-[13].

Among the available variants of the NOMA principle, power domain NOMA (PD-NOMA) has gained much research interest recently. In PD-NOMA, multiple users (in a user pair) share the same RB. Signal separation is performed in the power domain, by superimposing the signals of the users are superimposed and allocating different power levels to the signals associated to each set of paired users [11], [14], [15]. To recover the signal at the receiver's end, efficient interference cancellation techniques, such as successive interference cancellation (SIC), are used to the higher channel gain user, while direct signal recovery is applied to the lower channel gain user [10]. It has been reported in the literature that, through efficient user pairing and power allocation, NOMA can achieve higher capacity gains compared to conventional OMA schemes [16]. 
The integration of NOMA with other technologies, with the aim of further increasing the spectral efficiency of systems, has been a popular topic among researchers. Among the leading technologies for integration with PD-NOMA, device-to-device (D2D) communication is a particularly promising technique [17]-[19]. D2D communication is considered to have potential applications in the fifth generation (5G) to further improve the associated spectral efficiency. D2D communication enables low-power transmission of proximity services thus improving energy efficiency; it also allows the frequencies of cellular networks to be reused. Moreover, D2D communication may also facilitate new types of peer-to-peer (P2P) services. Furthermore, D2D communication can be extended research in combination with cognitive radio that is being studied recently [20]-[22]. In some works related to PD-NOMA with D2D, D2D-assisted PD-NOMA relay schemes were proposed to improve the signal-to-interference plus noise ratio (SINR) of low channel gain users in NOMA, while simultaneously enabling D2D communication. The main contributions of the current study are summarized as follows: The remainder of the paper is organized as follows. Section 2 presents the a model of the system being considered, the transmission protocol, and the signal-to-noise ratios (SNRs) received. A detailed ergodic capacity analysis is presented in Section 3 and Section 4 provides the simulation results and related discussions. Finally, Section 5 concludes the paper.

\section{RESEARCH METHOD}

This section presents a model of the system being considered and explains the transmission protocol.

a) The uplink NOMA system is investigated, where one of the two NOMA users simultaneously communicates with multiple other users/devices using D2D communication.

b) The ergodic sum capacity of the system is analyzed analytically, validated via simulations, and compared to that of conventional OMA and time-sharing NOMA systems.

c) The proposed model is demonstrated to achieve higher capacity gains than the OMA and time-sharing NOMA schemes, under scenarios where efficient interference cancellation can be ensured.

\subsection{System model}

Figure 1 depicts a model of the single-cell uplink transmission system consisting of a base station (BS), two cellular users $\left(U E_{1}, U E_{2}\right)$, and D2D users. $U E_{1}$ is located at the center of the cell, and $U E_{2}$ is located at the edge of the cell. Further, the $\mathrm{D} 2 \mathrm{D}$ users are located very close to $U E_{1}$ and $U E_{2}$. Each node is equipped with a single antenna. The channel coefficients for the links $U E_{1} \rightarrow \mathrm{BS}, U E_{2} \rightarrow \mathrm{BS}, U E_{1} \rightarrow D_{1 i}$, and $U E_{2} \rightarrow D_{2 i}$ are independent Rayleigh flat fading variables with channel coefficients $h_{1 b} \sim \mathcal{N}\left(0, \lambda_{1 b}=d_{1 b}^{-v}\right)$, $h_{2 b} \sim \mathcal{N}\left(0, \lambda_{2 b}=d_{2 b}^{-v}\right), \quad h_{1 i} \sim \mathcal{N}\left(0, \lambda_{1 i}=d_{1 i}^{-v}\right)$ and $h_{2 i} \sim \mathcal{N}\left(0, \lambda_{2 i}=d_{2 i}^{-v}\right)$ respectively, where $i \in$ $\{1,2, \cdots, N\} . v$ is the path loss exponent, and $d$ denotes distance. Moreover, as $d_{1 b}<d_{2 b}$ and $d_{11}<\cdots<$ $d_{1 i}<d_{1 b}$, it is expected that $\left|h_{1 b}\right|^{2}>\left|h_{2 b}\right|^{2}$ and $\left|h_{11}\right|^{2}>\cdots>\left|h_{1 i}\right|^{2}>\left|h_{1 b}\right|^{2}$. Furthermore, $d_{1 i}=d_{2 i}$ and $\left|h_{1 i}\right|^{2}=\left|h_{2 i}\right|^{2}$ are assumed for simplicity of the argument.

\subsection{Transmission protocol and received SNRs}

\subsubsection{Transmission protocol}

As shown in Figure 1, the cellular users $\left(U E_{1}, U E_{2}\right)$ transmit signals to the BS using the uplink NOMA principle. Moreover, when cellular users transmit signals to the BS, they simultaneously send the low power message signals to the more closely located D2D users using the downlink NOMA principle. Perfect successive interference cancellation (SIC) for NOMA signals is assumed not only at the BS but also for the D2D users. The transmission protocol is presented in detail as follows.

$U E_{1}$ transmits a superimposed composite signal $S_{1}=\sqrt{\alpha_{1} P} x_{1 b}+\sum_{k=1}^{N} \sqrt{\beta_{1 k} P} x_{1 k}$ to the BS and to $D_{1 i}$. Simultaneously, $U E_{2}$ transmits a superimposed composite signal $S_{2}=\sqrt{\alpha_{2} P} x_{2 b}+\sum_{k=1}^{N} \sqrt{\beta_{2 k} P} x_{2 k}$, where the first and second terms represent the $U E_{2} \rightarrow \mathrm{BS}$ and $U E_{2} \rightarrow D_{2 i}$ message signals, respectively. The parameters $\alpha_{1}, \alpha_{2}, \beta_{1 i}$, and $\beta_{2 i}$ are the power allocation coefficients for symbols, where $\alpha_{1}>\alpha_{2} \gg \beta_{1 N}=$ $\beta_{2 N}>\cdots>\beta_{11}=\beta_{21} \cdot \alpha_{1}$ and $\alpha_{2}$ are power allocation factors for the message signals from $U E_{1}$ and $U E_{2}$ to the BS, respectively. Moreover, the power allocation factors for the D2D users are $\beta_{1 i}$ and $\beta_{2 i}$ for $U E_{1} \rightarrow$ $D_{1 i}$ and $E_{2} \rightarrow D_{2 i}$, respectively. Their values are very small because of the following factors: (a) the very small distances between the cellular users and the D2D users, (b) small data rate requirements of the D2D users, and (c) the fact that larger values of $\beta_{1 i}$ and $\beta_{2 i}$ would cause interference for the cellular users and the BS. Furthermore, it is assumed that $U E_{1}$ and $U E_{2}$ cooperate with each other to adjust the power allocation factors for their signals. 


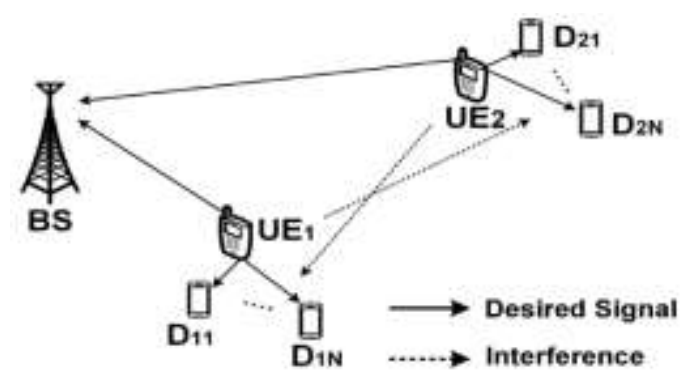

Figure 1. System model

\subsubsection{Received SNRs}

The term corresponding to the SNR received from a D2D user at the BS can be ignored because its value is negligible. This is because the power allocation factors for D2D users are very small. After receiving the superimposed signals, the BS first decodes the higher power signal $x_{1 b}$ from UE1 by treating the lower power signal $x_{2 b}$ of UE2 as noise. Subsequently, the BS performs SIC to decode the signal $x_{2 b}$. Thus, the SINRs received for the signals $x_{1 b}$ and $x_{2 b}$ at the BS are respectively denoted by:

$$
\begin{aligned}
& \gamma_{1 b}=\frac{\alpha_{1} \rho\left|h_{1 b}\right|^{2}}{\alpha_{2} \rho\left|h_{2 b}\right|^{2}+1} \\
& \gamma_{2 b}=\alpha_{2} \rho\left|h_{2 b}\right|^{2}
\end{aligned}
$$

where $\rho \triangleq \frac{P}{\sigma^{2}}$.

Meanwhile, when $D_{1 i}$ receives the superimposed signal, it performs SIC to obtain the signal $x_{1 i}$. The term corresponding to the signal transmitted from $U E_{2}$ to the $\mathrm{D} 2 \mathrm{D}$ user is assumed to be negligible due to channel attenuation. Therefore, the SINR received for the signal $x_{1 i}$ at $D_{1 i}$ is given by:

$$
\gamma_{1 i}=\frac{\beta_{1 i} \rho\left|h_{1 i}\right|^{2}}{\sum_{k=1}^{i-1} \beta_{1 k} \rho\left|h_{1 k}\right|^{2}+1}
$$

Likewise, the SINR received for the signal $x_{2 i}$ at $D_{2 i}$ is given by:

$$
\gamma_{2 i}=\frac{\beta_{2 i} \rho\left|h_{2 i}\right|^{2}}{\sum_{k=1}^{i-1} \beta_{2 k} \rho\left|h_{2 k}\right|^{2}+1}
$$

\section{ERGODIC CAPACITY ANALYSIS}

This section investigates the achievable capacity of individual signal, and then computes the overall ergodic sum capacity of the system [23].

\subsection{Ergodic capacity of $U E_{1}$}

Using (1), the achievable capacity associated to the signal $x_{1 b}$, is given by:

$$
C_{x_{1 b}}=\log _{2}\left(1+\gamma_{1 b}\right)
$$

Let $X_{1 b}=\frac{\alpha_{1} \rho\left|h_{1 b}\right|^{2}}{\alpha_{2} \rho\left|h_{2 b}\right|^{2}+1}$. As in (6), the cumulative distribution function (CDF) of $X_{1 b}, F_{X_{1 b}}(x)$ can be written as follows.

$$
F_{X_{1 b}}(x)=1-\left(\frac{\alpha_{1} \rho \lambda_{1 b}}{\alpha_{1} \rho \lambda_{1 b}+\alpha_{2} \rho \lambda_{2 b} x}\right) e^{-\frac{x}{\alpha_{1} \rho \lambda_{1 b}}}
$$

Using the identity $\int_{0}^{\infty} \log _{2}(1+x) f_{X}(x) d x=\frac{1}{\ln 2} \int_{0}^{\infty} \frac{1-F_{X}(x)}{1+x} d x$, the ergodic capacity (EC) $\bar{C}_{x_{1 b}}$ of $x_{1 b}$ is computed as in (7). 


$$
\bar{C}_{x_{1 b}}=\frac{1}{\ln 2} \int_{0}^{\infty} \frac{1}{1+x}\left(\frac{\alpha_{1} \rho \lambda_{1 b}}{\alpha_{1} \rho \lambda_{1 b}+\alpha_{2} \rho \lambda_{2 b} x}\right) e^{-\frac{x}{\alpha_{1} \rho \lambda_{1 b}}} d x
$$

Using $\operatorname{Ei}(u)=\int_{-\infty}^{u} \frac{e^{x}}{x} d x, u<0$, where Ei represents the exponential integral function [24] equation (3.352.4), the ergodic capacity $\bar{C}_{x_{1 b}}$ is computed as follows.

$$
\begin{aligned}
& \bar{C}_{x_{1 b}}=-\frac{1}{\ln 2\left(1-\frac{\alpha_{2} \rho \lambda_{2 b}}{\alpha_{1} \rho \lambda_{1 b}}\right)}\left\{e^{\frac{1}{\alpha_{1} \rho \lambda_{1 b}}} E i\left(-\frac{1}{\alpha_{1} \rho \lambda_{1 b}}\right)-e^{\frac{1}{\alpha_{2} \rho \lambda_{2 b}}} E i\left(-\frac{1}{\alpha_{2} \rho \lambda_{2 b}}\right)\right\}=-\frac{1}{\ln 2\left(1-\frac{q}{p}\right)}\left\{e^{\frac{1}{p}} E i\left(-\frac{1}{p}\right)-\right. \\
& \left.e^{\frac{1}{q}} E i\left(-\frac{1}{q}\right)\right\}
\end{aligned}
$$

where $p=\alpha_{1} \rho \lambda_{1 b}$, and $q=\alpha_{2} \rho \lambda_{2 b}$.

\subsection{Ergodic capacity of $U E_{2}$}

Using (2), the achievable capacity associated to the signal $x_{2 b}$ is given by:

$C_{x_{2 b}}=\log _{2}\left(1+\gamma_{x_{2 b}}\right)$

Let $X_{2 b}=\gamma_{x_{2 b}}=\alpha_{2} \rho\left|h_{2 b}\right|^{2}$. The CDF of $X_{2 b}, F_{X_{2 b}}(x)$ can be written as follows

$F_{X_{2 b}}(x)=1-e^{-\frac{x}{\alpha_{2} \rho \lambda_{2 b}}}$

Similar to the calculation of $\bar{C}_{x_{1 b}}, \bar{C}_{x_{2 b}}$ can be computed as follows.

$\bar{C}_{x_{2 b}}=\frac{1}{\ln 2} \int_{0}^{\infty} \frac{1}{1+x} e^{-\frac{x}{\alpha_{2} \rho \lambda_{2 b}}} d x$

Using $\operatorname{Ei}(u)=\int_{-\infty}^{u} \frac{e^{x}}{x} d x, u<0, \bar{C}_{x_{2 b}}$ of $x_{2 b}$ is computed as follows.

$\bar{C}_{x_{2 b}}=-\frac{1}{\ln 2}\left\{e^{\frac{1}{\alpha_{2} \rho \lambda_{2 b}}} E i\left(-\frac{1}{\alpha_{2} \rho \lambda_{2 b}}\right)\right\}=-\frac{1}{\ln 2}\left\{e^{\frac{1}{q}} E i\left(-\frac{1}{q}\right)\right\}$

where $q=\alpha_{2} \rho \lambda_{2 b}$, exactly as defined earlier in the case of the UE1 ergodic capacity.

\subsection{Ergodic capacity of $D_{11}$}

Using (3), the achievable capacity associated to the signal $x_{11}$ is given by:

$C_{x_{11}}=\log _{2}\left(1+\gamma_{11}\right)$

Let $Y_{11}=\gamma_{11}=\beta_{11} \rho\left|h_{11}\right|^{2}$. The CDF of $Y_{11}, F_{Y_{11}}(y)$ can be written as follows.

$F_{Y_{11}}(y)=1-e^{-\frac{y}{\beta_{11} \rho \lambda_{11}}}$

Similar to (7) and (8), the ergodic capacity $\bar{C}_{x_{11}}$ of $x_{11}$ can be computed as follows.

$$
\begin{aligned}
& \bar{C}_{x_{11}}=\frac{1}{\ln 2} \int_{0}^{\infty} \frac{1}{1+y} e^{-\frac{y}{\beta_{11} \rho \lambda_{11}}} d y=-\frac{1}{\ln 2}\left\{e^{\frac{1}{\beta_{11} \rho \lambda_{11}}} E i\left(-\frac{1}{\beta_{11} \rho \lambda_{11}}\right)\right\} \\
& =-\frac{1}{\ln 2}\left\{e^{\frac{1}{r}} E i\left(-\frac{1}{r}\right)\right\}
\end{aligned}
$$

where $r=\beta_{11} \rho \lambda_{11}$ 


\subsection{Ergodic capacity of $D_{12}$}

Using (4), the achievable capacity associated to the signal $x_{12}$ is given by:

$$
C_{x_{12}}=\log _{2}\left(1+\gamma_{12}\right)
$$

Let $Y_{12}=\frac{\beta_{12} \rho\left|h_{12}\right|^{2}}{\beta_{11} \rho\left|h_{12}\right|^{2}+1}$ can be written as

$$
F_{Y_{12}}(y)=1-e^{-\frac{y}{\left(\beta_{12}-\beta_{11} y\right) \rho \lambda_{12}}}
$$

According to [25], it is quite difficult to obtain a closed-form solution for functions such as $F_{Y_{12}}(y)$. Hence, by applying a high SNR approximation $(\rho \rightarrow \infty), \gamma_{12}$ can be approximated as $\gamma_{12}=\frac{\beta_{12}}{\beta_{11}}$. The ergodic capacity of $D_{12}$ is

$$
\bar{C}_{x_{12}}^{a p p r}=\log _{2}\left(1+\frac{\beta_{12}}{\beta_{11}}\right)
$$

\subsection{Conventional multiple access}

For comparison with proposed scheme, an OMA scheme and a time-sharing based NOMA are devised as benchmarks. First, an OMA scheme assumes that the frequency is properly divided and transmitted. Let $B(0 \leq B \leq 1)$ denote the bandwidth used. In the model of the system assumed in this paper, $\frac{1}{6} B$ is allocated to each cellular user and each D2D user. Secondly, time-sharing based NOMA assumes that time is divided into three phases. In the first phase, the cellular users transmit signals to the BS simultaneously. In the second phase, UE1 transmits signals to $D_{11}$ and $D_{12}$. In the third phase, UE2 transmits signals to $D_{21}$ and $D_{22}$.

\subsection{Ergodic capacities of $D_{21}$ and $D_{22}$} computed as:

Similar to the calculations of $\bar{C}_{x_{11}}$ and $\bar{C}_{x_{12}}^{a p p r}$, the ergodic capacities of $\bar{C}_{x_{21}}$ and $\bar{C}_{x_{22}}^{a p p r}$ can be

$$
\begin{aligned}
& \bar{C}_{x_{21}}=-\frac{1}{\ln 2}\left\{e^{\frac{1}{\beta_{21} \rho \lambda_{21}}} \operatorname{Ei}\left(-\frac{1}{\beta_{21} \rho \lambda_{21}}\right)\right\} \\
& \bar{C}_{x_{22}}^{a p p r}=\log _{2}\left(1+\frac{\beta_{22}}{\beta_{21}}\right)
\end{aligned}
$$

\subsection{Ergodic sum capacity}

Using (8), (12), (15), and (20), the ergodic sum capacity of the considered NOMA-D2D system model can be calculated by (21).

$$
\bar{C}_{\text {sum }} \sim \bar{C}_{x_{1 b}}+\bar{C}_{x_{2 b}}+\bar{C}_{x_{11}}+\bar{C}_{x_{12}}^{a p p r}+\bar{C}_{x_{21}}+\bar{C}_{x_{22}}^{a p p r}
$$

where the first two terms correspond to the signals transmitted by the cellular users to the BS, and the other terms correspond to the D2D communication.

\section{RESULTS AND DISCUSSION}

This section presents the simulation and the analytical results of the considered NOMA-D2D system model. A close agreement between the simulation and the analytical results validates the mathematical analysis. Moreover, the ergodic sum capacity of the NOMA-D2D system model being considered is compared with that of the conventional OMA scheme and the time-sharing-based NOMA. It is shown that, under efficient interference cancellation, the NOMA-D2D system demonstrates higher capacity gains than the conventional benchmark schemes. The simulation is performed using MATLAB R2016b.

Considering the simulation setup, various system parameters are detailed as follows. The normalized distances of cellular users UE1 and UE2 from the BS are maintained as $d_{1 b}=0.3$ and $d_{2 b}=1$, respectively. Moreover, the D2D devices $D_{11}, D_{12}, D_{21}$, and $D_{22}$ are at distances $d_{11}=d_{21}=0.05$, and $d_{12}=d_{22}=0.1$ away from UE1 and UE2 respectively. The path loss factor is $v=4$. Furthermore, the power allocation coefficients are set as $\alpha_{1}=0.64, \alpha_{2}=0.24, \beta_{11}=\beta_{21}=0.01$, and $\beta_{21}=\beta_{22}=0.05$. 
Figure 2 provides a comparison of the ergodic sum capacities of the NOMA-D2D system and the conventional OMA and the time-sharing-based NOMA with respect to the transmitted SNRs. The analytical and simulated results of NOMA-D2D system model are presented. A close concordance between the results validates the presented mathematical analysis. As shown, the ergodic sum capacity of NOMA-D2D shows a remarkable performance gain over that of the OMA scheme under perfect SIC assumptions. For instance, when $\rho=20 \mathrm{~dB}$, NOMA-D2D achieves capacity gains of $192.2 \%$ and $157.5 \%$ over the conventional OMA and the time-sharing-based NOMA, respectively.

Figure 3 presents the ergodic capacity of each user under the scenario of perfect interference cancellation. Both analytical and simulated results are plotted, and a close agreement between the results validates the mathematical analysis. As is evident from the results, the capacity of $D_{11}$ is the highest at the all-transmitted SNR values. This is because $D_{11}$ is located very close to UE1. It can be argued that the channel corresponding to $D_{11}$ is in very good condition. The results for scenarios with imperfect interference cancellation are not presented for individual users. However, it is well known that, if imperfections were to occur in the interference cancellation and SIC, the capacities of UE2 and $D_{11}$ would further decrease.

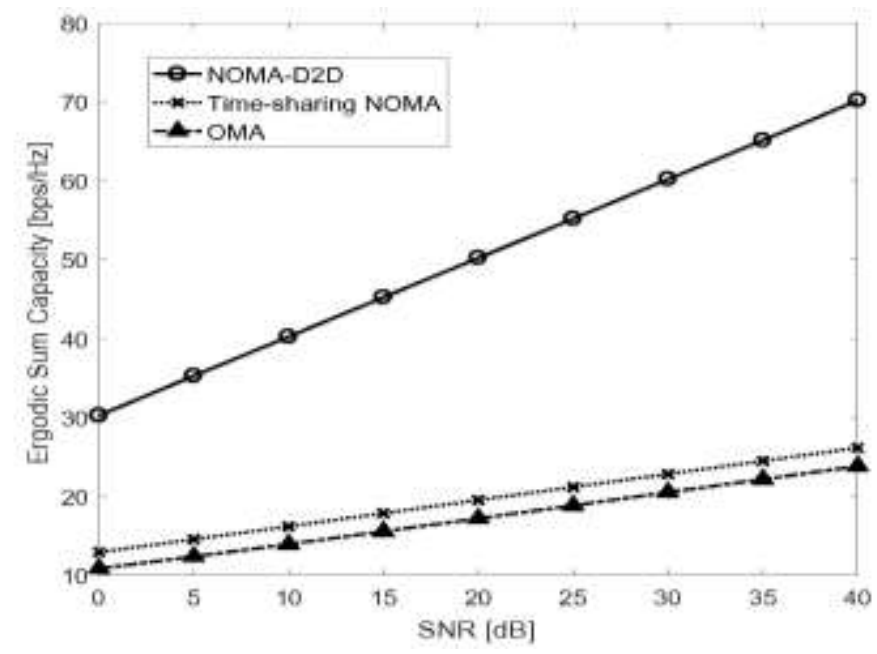

Figure 2. Ergodic sum capacity analysis

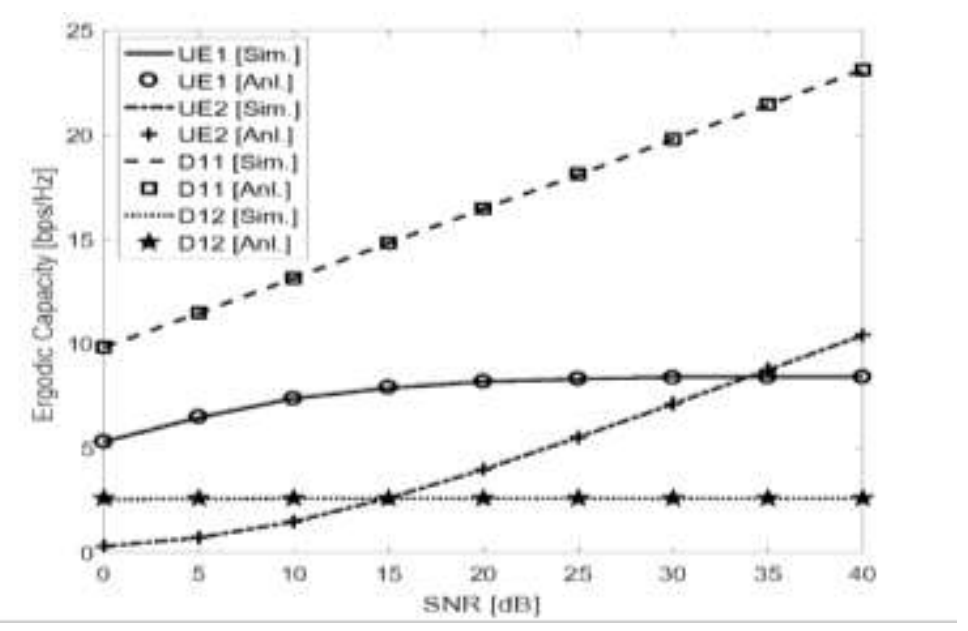

Figure 3. Ergodic capacity analysis

\section{CONCLUSION}

In this paper, an uplink communication system that integrates NOMA with D2D communication has been investigated. In the NOMA-D2D system, two cellular users communicated with the BS using uplink NOMA, and two cellular users simultaneously communicated with the D2D users using downlink NOMA. 
Comprehensive analytical derivations were presented to compute the ergodic sum capacity of the model of the investigated system. The mathematical analysis was validated through simulations, where a close agreement between the analytical and simulated results was observed. Capacity comparisons have been presented. The proposed NOMA-D2D system outperformed the conventional OMA and the time-sharingbased NOMA in the perfect interference cancellation scenario. Future works may focus on the generalization and downlink of the NOMA-D2D. The multi-cell case also can be considered.

\section{ACKNOWLEDGEMENTS}

This work was supported by the National Research Foundation of Korea (NRF) grant funded by the Korea government. (MSIT) (No. 2019R1A2C1089542).

\section{REFERENCES}

[1] Y. Saito, Y. Kishiyama, A. Benjebbour, T. Nakamura, A. Li, and K. Higuchi, "Non-Orthogonal Multiple Access (NOMA) for Cellular Future Radio Access," 2013 IEEE 77th Vehicular Technology Conference (VTC Spring), 2013, pp. 1-5, doi: 10.1109/VTCSpring.2013.6692652

[2] W. Liang, Z. Ding and H. V. Poor, "Non-orthogonal multiple access (NOMA) for 5G systems," in Key Technologies for 5G Wireless Systems, Cambridge, U.K.:Cambridge Univ. Press, 2017, pp. 109-132.

[3] D. Bega, M. Gramaglia, C. J. B. Cano, A. Banchs, and X. Costa-Perez, "Toward the Network of the future: From Enabling Technologies to 5G concepts," Transactions on Emerging Telecommunications Technologies, vol. 28, no. 8, 2017, doi: 10.1002/ett.3205

[4] Z. Yang, W. Xu, Y. Pan, C. Pan, and M. Chen, "Energy Efficient Resource Allocation in Machine-to-Machine Communications with Multiple Access and Energy Harvesting for IoT," in IEEE Internet of Things Journal, vol. 5, no. 1, pp. 229-245, Feb. 2018, doi: 10.1109/JIOT.2017.2778766

[5] L. Pei et al., "Energy-Efficient D2D Communications Underlaying NOMA-Based Networks with Energy Harvesting," in IEEE Communications Letters, vol. 22, no. 5, pp. 914-917, May 2018, doi: 10.1109/LCOMM.2018.2811782

[6] M. Basharat, W. Ejaz, M, Naeem, A. M.Khattak, and A. Anpalagan, "A Survey and Taxonomy on Nonorthogonal Multiple-Access Schemes for 5G Networks," Transactions on Emerging Telecommunications Technologies, vol. 29, no. 1, 2018, doi: 10.1002/ett.3202

[7] Z. Ding et al., "Application of Non-Orthogonal Multiple Access in LTE and 5G Networks," in IEEE Communications Magazine, vol. 55, no. 2, pp. 185-191, February 2017, doi: 10.1109/MCOM.2017.1500657CM

[8] Y. Liu, Z. Qin, M. Elkashlan, Z. Ding, A. Nallanathan, and L. Hanzo, "Nonorthogonal Multiple Access for 5G and Beyond," in Proceedings of the IEEE, vol. 105, no. 12, pp. 2347-2381, Dec. 2017, doi: 10.1109/JPROC.2017.2768666

[9] Z. Ding, X. Lei, G. K. Karagiannidis, R. Schober, J. Yuan, and V. K. Bhargava, "A Survey on Non-Orthogonal Multiple Access for 5G Networks: Research Challenges and Future Trends," in IEEE Journal on Selected Areas in Communications, vol. 35, no. 10, pp. 2181-2195, Oct. 2017, doi: 10.1109/JSAC.2017.2725519

[10] K. Saito, A. Benjebbour, Y. Kishiyama, Y. Okumura, and T. Nakamura, "Performance and Design of SIC Receiver for Downlink NOMA with Open-Loop SU-MIMO," 2015 IEEE International Conference on Communication Workshop (ICCW), 2015, pp. 1161-1165, doi: 10.1109/ICCW.2015.7247334

[11] Z. Ding, P. Fan, and H. V. Poor, "Impact of User Pairing on 5G Nonorthogonal Multiple-Access Downlink Transmissions," in IEEE Transactions on Vehicular Technology, vol. 65, no. 8, pp. 6010-6023, Aug. 2016, doi: 10.1109/TVT.2015.2480766

[12] M. B. Shahab, M. F. Kader, and S. Y. Shin, "A Virtual User Pairing Scheme to Optimally Utilize the Spectrum of Unpaired Users in Non-orthogonal Multiple Access," in IEEE Signal Processing Letters, vol. 23, no. 12, pp. 17661770, Dec. 2016, doi: 10.1109/LSP.2016.2619371

[13] M. B. Shahab and S. Y. Shin, "Time Shared Half/Full-Duplex Cooperative NOMA with Clustered Cell Edge Users," in IEEE Communications Letters, vol. 22, no. 9, pp. 1794-1797, Sept. 2018, doi: 10.1109/LCOMM.2018.2853627

[14] J. Choi, "On the Power Allocation for a Practical Multiuser Superposition Scheme in NOMA Systems," in IEEE Communications Letters, vol. 20, no. 3, pp. 438-441, March 2016, doi: 10.1109/LCOMM.2016.2518165

[15] M. B. Shahab and S. Y. Shin, "User Pairing and Power Allocation for Non-Orthogonal Multiple Access: Capacity Maximization Under Data Reliability Constraints," Physical Communication, vol. 30, pp. 132-144, 2018, doi: 10.1016/j.phycom.2018.05.010

[16] M. Zeng, A. Yadav, O. A. Dobre, G. I. Tsiropoulos, and H. V. Poor, "Capacity Comparison Between MIMONOMA and MIMO-OMA with Multiple Users in a Cluster," in IEEE Journal on Selected Areas in Communications, vol. 35, no. 10, pp. 2413-2424, Oct. 2017, doi: 10.1109/JSAC.2017.2725879.

[17] J. Zhao, Y. Liu, K. K. Chai, Y. Chen, M. Elkashlan, and J. Alonso-Zarate, "NOMA-Based D2D Communications: Towards 5G," 2016 IEEE Global Communications Conference (GLOBECOM), 2016, pp. 1-6, doi: 10.1109/GLOCOM.2016.7842024

[18] J. Zhao, Y. Liu, K. K. Chai, Y. Chen, and M. Elkashlan, "Joint Subchannel and Power Allocation for NOMA Enhanced D2D Communications," in IEEE Transactions on Communications, vol. 65, no. 11, pp. 5081-5094, Nov. 2017, doi: 10.1109/TCOMM.2017.2741941 
[19] Y. B. Song, H. S. Kang, and D. K. Kim, "5G cellular systems with D2D assisted NOMA relay," 2016 URSI AsiaPacific Radio Science Conference (URSI AP-RASC), 2016, pp. 1-3, doi: 10.1109/URSIAP-RASC.2016.7883540

[20] M. Cicioğlu, M. E. Bayrakdar, and A. Çalhan, "Performance Analysis of A New Mac Protocol for Wireless Cognitive Radio Networks," Wireless Personal Communications, vol. 108, no. 1, pp. 67-86, 2019, doi: 10.1007/s11277-019-06388-w

[21] M. E. Bayrakdar, "Cooperative Communication Based Access Technique for Sensor Networks," International Journal of Electronics, vol. 107, no. 2, pp. 212-225, 2020, doi: 10.1080/00207217.2019.1636313

[22] M. E. Bayrakdar, "Exploiting cognitive wireless nodes for priority-based data communication in terrestrial sensor networks," ETRI Journal, vol. 42, no. 1, pp. 36-45, 2020, doi: 10.4218/etrij.2019-0296

[23] P. Varzakas, "Average Channel Capacity for Rayleigh Fading Spread Spectrum Mimo Systems," International Journal of Communication Systems, vol. 19, no. 10, pp. 1081-1087, 2006, doi: 10.1002/dac.784.

[24] I. S. Gradshteyn and I. M. Ryzhik, Table of Integrals, Series, and Products. 7th edn. New York, NY, USA: Academic Press, 2007.

[25] J. Kim and I. Lee, "Non-Orthogonal Multiple Access in Coordinated Direct and Relay Transmission," in IEEE Communications Letters, vol. 19, no. 11, pp. 2037-2040, Nov. 2015, doi: 10.1109/LCOMM.2015.2474856

\section{BIOGRAPHIES OF AUTHORS}

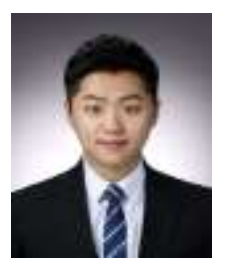

Sang Hoon Lee received his B.S. in electronic engineering from Kumoh National Institute of Technology Gumi, Republic of Korea on 2013. He received his M.S degrees in IT convergence engineering from Kumoh National Institute of Technology on 2015. His research areas are future radio access, and wireless communication systems.

Further info on his homepage: http://wens.re.kr

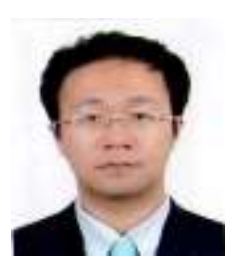

Soo Young Shin received his Ph.D. degrees in electrical engineering and computer science from Seoul National University on 2006. He was with WiMAX Design Lab, Samsung Electronics, Suwon, South Korea from 2007 to 2010. He joined as full-time professor to School of Electronics, Kumoh National Institute of Technology, Gumi, South Korea from 2010. He is currently an Associate Professor. He was a post Doc. researcher at University of Washington, USA at 2007. In addition, he was a visiting scholar to University of the British Columbia, Canada at 2017. His research interests include 5G/6G wireless communications and networks, signal processing, Internet of things, mixed reality, and drone applications.

Further info on his homepage: http://wens.re.kr 